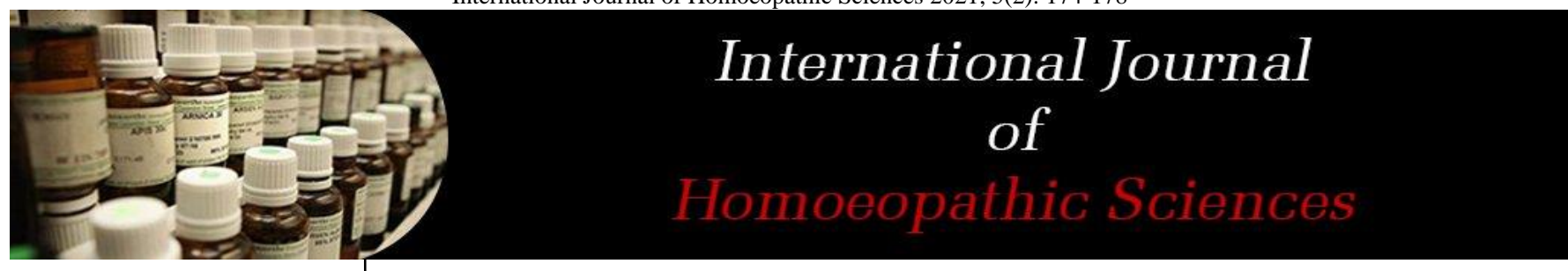

E-ISSN: 2616-4493 P-ISSN: 2616-4485 www.homoeopathicjournal.com IJHS 2021; 5(2): 174-178 Received: 25-02-2021 Accepted: 27-03-2021

Dr. Hozaifa Ayubi PG Scholar, Department Practice of Medicine, RBTS Government Homoeopathic Medical College and Hospital, Muzaffarpur, Bihar, India

Dr. Rajani Kumari PG Scholar, Department Practice of Medicine, RBTS Government Homoeopathic Medical College and Hospital, Muzaffarpur, Bihar, India
Corresponding Author: Dr. Hozaifa Ayubi PG Scholar, Department Practice of Medicine, RBTS Government Homoeopathic Medical College and Hospital, Muzaffarpur, Bihar, India

\section{Homoeopathy a boon for rheumatoid arthiritis}

\author{
Dr. Hozaifa Ayubi and Dr. Rajani Kumari
}

DOI: $\underline{\text { https://doi.org/10.33545/26164485.2021.v5.i2c.381 }}$

\begin{abstract}
Bone is the important part of our body, without bone there is no existence of human body. Bone is the frame of a human body; The whole-body structure depends upon bone. Any disease related to bone and related structure will lead to one of the leading disorders of bone that is RHEUMATOID ARTHIRITIS. Rheumatoid Arthritisis an auto-immune enlightened disorder of cartilage degeneration, inflammation, and affects joint lining causing painful swelling. Rheumatoid Arthritis leads to pain, disability as well as difficulty in joints and restricts the routine movements of human being. Rheumatoid Arthritis is most common rheumatic disease; Limitation of controversial medicine management of this condition indicates the real need to safe and effective treatment of osteoarthritis. In Homoeopathy there is lots of scope for the treatment of this disease because homoeopathy follows the nature of law similia similibus curenture and treats the person as a whole by doing the individualization and selecting the constitutional medicine and stops the recurrence of auto-immune diseases like Rheumatoid Arthritis, psoriasis and others without other suffering and side effects.
\end{abstract}

Keywords: homoeopathy, rheumatoid arthritis, auto-immune, management, miasm

\section{Introduction}

The name is derived from the Greek rheumatic meaning "flowing", the suffix -oid meaning "in the shape of", arthr meaning "joint" and the suffix -it is, a "condition involving inflammation". Rheumatoid arthritis (RA) is traditionally considered a chronic, inflammatory autoimmune disorder that causes the immune system to attack the joints. It is a disabling and painful inflammatory condition, which can lead to substantial loss of mobility due to pain and joint destruction. RA is a systemic disease, often affecting extra-articular tissues throughout the body including the skin, blood vessels, heart, lungs, and muscles. Rheumatoid arthritis now become the most prevalent disease in our society and generates enormous costs of health. Pain, Swelling, tenderness, stiffness and degeneration are common symptoms of Rheumatoid arthritis while constitutional symptoms as malaise, fatigue, loss of appetite anxiety and depression are associated with this condition.

The main aims of conventional therapy of Rheumatoid arthritis are to control the pain and reduction of progressive of joint damage in order to minimize disability and maximize quality of life. In additional drug therapy includes non-opioid analgesics such as paracetamol, non-steroidal anti-inflammatory drug (NASAIDs) topical analgesic opioid analgesics and intra articular steroid injection such treatments may prove ineffectual in some patients and NSAIDs are potentially toxic serious adverse effects. There is a need for such medicines by patients who do not respond well to conventional medical therapy and who are not suitable for or refuse surgery. Many patients increasingly choosing the complementary or alternative medicines (CAM). The efficacy of CAM by suffers of rheumatic disease is highly prevalent and increasing, with homoeopathy being one of the most popular CAM treatments in this patient's group. Belief in the effectiveness of homoeopathy in general is widespread and growing among physicians and the public.

Homoeopathy has got a very remarkable result and is very helpful in treating cases of Rheumatoid arthritis with no side effects. Pain, discomfort, restricted movement all are well treated with homoeopathy medicine. Homoeopathic medicine can act marvelously before and after surgical management. Other types of arthritis are well cured by homoeopathy.

\section{Epidemiology}

The incidence of RA is 30 cases per 10,000 populations. The peak incidence is between the ages of 40 and 60 . The prevalence rate is $1 \%$, with women affected three to five times as often as men. 
Some Native American groups have higher prevalence rates (5-6\%) and black persons from the Caribbean region have lower prevalence rates. First-degree relatives' prevalence rate is $2-3 \%$ and disease concordance in monozygotic twins is approximately $15-20 \%$.

Rheumatoid arthritis occurs most frequently in the 40-60 age group, although can start at any age. It is strongly associated with the HLA marker DR4 (W4, W14 \& W15 are associated with the disease and W10 \& W13 are protective). Hence family history is an important risk factor. The disease is 3 times more common in women than men and up to 4 times more common in smokers than non-smokers.

\section{Rheumatic arthiritis}

Definition: Rheumatoid arthritis is a common chronic, systemic disease, producing:

1. A systemic inflammatory polyarthritis

2. Extra-articular involvement, e.g. in the lung and many other organs

3. Progressive joint damage causing severe disability

\section{Aetiology}

Auto-immunity is held responsible for this disease.

\section{Pathology}

There are two main pathological characteristics inflammation and proliferation.

The synovium shows signs of a chronic inflammatory reaction, with infiltration of lymphocytes, plasma cells and macrophages. It then proliferates and grows out over the surface of the cartilage, producing a tumor like mass called "pannus."

\section{Symptoms}

a. Joint pain: the pain is worst on waking in the morning and may improve with activity. There is often pain at night and disturbed sleep

b. Morning stiffness: often lasting for several hours

c. General symptoms: fatigue and general malaise are common

d. Disability: depends upon the change in the individual joints.

\section{Signs}

a. Swelling: soft tissue swelling caused by effusion

b. Warmth

c. Tenderness on pressure and on movement

d. Limitation of movement with muscle wasting around the affected joint

e. Deformities occurring in the latter stage of the disease.

\section{Common joint deformities in RA}

- Swan-neck deformity

- Boutonnière deformity

- Ulnar deviation of fingers

\section{Non-articular features}

\section{A. Soft tissue surrounding joints}

1. Rheumatoid nodules are found in about $20 \%$ of cases. They are most often felt on the ulnar surface of the forearm below elbow. Patients with nodules are usually sero-positive.

2. Bursitis: The olecranon and other bursae may be swollen.
3. Tenosynovitis: Particularly affecting the flexor tendon in the palm of the hand.

4. Muscle wasting around the affected joint.

\section{B. The eyes}

1. The commonest eye problem in RA is secondary Sjogren' syndrome in 15\% cases (Kerato-conjunctivitis sicca), a dry mouth (xerostomia) and RA

2. Scleritis may be occur, causing a painful red eye

\section{The nervous system}

1. Carpal tunnel syndrome is the commonest

2. Poly neuropathy occurs rarely causing glove and stoking sensory loss and sometime motor weakness

\section{The spleen, lymph nodes and blood}

1. Palpable lymph nodes are common, usually in the distribution of the affected joint

2. The spleen may be enlarged. RA with splenomegaly and neutropenic is known as Fealty's syndrome

3. Anemia: Usually normochromic and normocytic anemias is seen

4. Thrombocytosis may be occurred

\section{E. The lungs}

1. Pleural effusion: commonest, the fluid has a high protein and low sugar content

2. Rheumatoid nodules in the lungs can be up to $3 \mathrm{~cm}$ in diameter

3. Small airway disease is commoner in patient with RA smoke than in normal people who do not smoke

\section{F. The heart}

A pericardial rub is often heard in patient with RA.

\section{G. The kidneys}

RA is a common cause of amyloidosis affecting the kidney. It usually presents as proteinuria and may go on to renal failure or to the nephritic syndrome.

\section{Classification of disease severity in RA}

Class I: No restriction of ability to perform normal activity. Class II: Moderate restriction, but adequate for normal activities.

Class III: Marked restriction, inability to perform most duties related to occupation of self-care.

Class IV: Incapacitation or confinement to wheel chair or bed.

\section{Investigations}

\section{Hematological studies}

- TC

- $\mathrm{DC}$

- Leucopenia, especially neutropenia, is found in Felty's syndrome

- Eosinophilia suggests vasculitis

- ESR generally elevated

- $\mathrm{Hb} \%$ mild form anemia mostly normocytic, normochromic

- C-reactive protein are elevated

\section{Biochemical test}

- Plasma albumin to globulin ratio may be altered.

- Liver function taste- active disease is associated with a 
slight increase in the alkaline phosphate.

- Hyperuricemia may be present.

3. Microbiological test: Synovial fluid culture is always necessary when arthrocentesis is carried out because there may be co-existent infection.

\section{Serological}

- Rheumatoid factor (RF).

- The high titer of RF is expected to be present. But the presence of RF is not specific for RA.

- Antinuclear antibodies:

Antinuclear antibodies are demonstrable in $20 \%$ of patient.

\section{Radiological findings}

It shows characteristic changes are symmetrical pattern of involvement

- Soft tissue swelling

- Joint space narrowing

- Marginal erosion

- Mouse eaten appearance due to erosion

- Joint deformity

- Bone end may show osteoporosis and cyst may be develop

\section{Synovial fluid analysis}

- Rheumatoid synovial fluid is cloudy and often green tinged

- The white cell count varies between 5-50000 cells pr micro litter.

- Synovial fluid glucose is often reduced.

\section{Diagnosis}

In 1797, the American college of Rheumatology developed revise criteria for the classification of Rheumatoid arthritis. These are following:

\section{Guideline for classification}

Four or seven criteria are required to classify a patient as having rheumatoid arthritis

\section{Criteria}

a. Morning stiffness: stiffness in and around the joint lasting one hour before maximal improvement

b. Arthritis of three or more joint areas, at least three joint areas observed by a physician simultaneously, have soft tissue swelling or joint effusion.

c. Arthritis of hand joint; arthritis of wrist, metacarpophalangeal joint or proximal inter-phalangeal joint

d. Symmetric arthritis; simultaneous involvement of the same joint areas on both sides of the body

e. Rheumatoid nodules

f. Serum rheumatoid factor: demonstration of abnormal amounts of serum rheumatoid factor

g. Radiographic changes

Among these, criteria a-d must be present for at least 6 weeks, criteria b-e must be observed by a physician.

\section{Management}

In the management of RA, the goals of the physician are:

1. Relief of pain.

2. Reduction of inflammation.

3. Protection of articular structures.
4. Maintenance of function.

5. Control of systemic involvement.

To achieve these following procedures are taken

a. Systemic rest.

b. Articular support.

c. Exercise:

\section{It may be with either of two main objectives}

- To maintain or improve the range of joint movement.

- Increase muscle strength and improve joint stability.

d. Heat and cold: radiant or moist heat is generally most satisfactory. Some patient derives more relief from joint pain from local application of cold.

e. Hydrotherapy: It can use to

- Reduce pain.

- Increase range of movement.

- Increase muscle power.

f. Support and stabilization of joint.

g. Weight loss.

h. Diet.

\section{Homoeopathic concept of rheumatoid arthritis}

Homoeopathy recognized no such method of treatment as prescribing for any diagnostic entity by name.

According to Homoeopathic system of medicine disease is primarily only on altered state of life and mind, manifesting itself on morbid functions and sensation. To a homoeopathic physician the totality of the morbid functions and sensation of one patient is the disease. The totality of symptoms constitutes the true and only conceivable portrait of the disease.

Totality of symptoms should be considered in the treatment of a patient, which is the only guide of the homoeopathic treatment.

Classification of the diseases by Hahnemann is unique, rational and clinical one. His classification is based on many clinical criteria which will remain unchanged forever. They are divided into acute and chronic diseases. Acute diseases are generally only a transient explosion of latent psora whereas true chronic diseases are caused by a chronic miasm.

Rheumatoid arthritis is a degenerative disease. Degenerative diseases are always outcome of active miasmatic mixtures. The miasmatic diversification of the human mind, together with its civilization' brings on a condition favoring a degenerative disease.

Psora is a perturbation of nutrition. Sycosis compels it to accumulate debris which should be eliminated, and syphilis cause it to degenerate. Since all of these conditions can act as the true causes of all imbalances - both dynamic and somatic. Each one of them, and A PRIORI their mixtures, is a sufficient etiology for every degenerative disease.

\section{Organon of Medicine}

According to organon of medicine, Aphorism-67 (FN) only in the most urgent cases, where danger to life and imminent death allow no time for the action of a homoeopathic remedy...In sudden accident.

While treating rheumatoid artihiritis in homoeopathy we may divide in 2 category-

1. Surgical

2. Non-surgical 
Sec-189-and yet very little reflection will suffice to convince us the no external malady can arise, ..., the cooperation of the whole organism... no eruption on lip, no whitlow can occur without previous and simultaneous internal ill-health.

Sec-193- by means of medicine employed internally... The general morbid state of the body is removed along with local affection... as one of the most considerable and....

Sec-204-if we deduct all chronic affection, ailments and disease that depends on [persistent unhealthy mode of living...

Rheumatoid arthritis: A miasmatic view from the standpoint of clinical feature

Psora

- Neuralgic pains are usually relieved by quiet, rest and warmth, worse by motion.

- Cramps in the lower extremities in the calves of the legs, in the feet toes, ankles.

- Numbness of the extremities with tingling in the fingers.

\section{Sycosis}

- Shooting and tearing pains in the muscles and the joints, pain in fingers or small joints.

- The sycotic pains are relieved by rest and the patient is relieved by moving, by rubbing, stretching, and better in dry, fair, weather.

- Pain worse at the approach of a storm or a damp, humid atmosphere and becoming cold.

- Stiffness and soreness, especially lameness, is very characteristic of sycosis.

- Infiltration of inflammatory deposits, but it readily absorbs and never formative.

- It produces violent palpitation with beating of the whole body from reflex rheumatic trouble.

\section{Syphilis}

- Stitching, shooting or lancinating pains in the periosteum or long bones of the upper or lower extremities.

- Pains worse at night, or at the approach of the night. They are also worse by change of weather, by cold and damp atmosphere.

- Nodular growth.

- Infiltration of inflammatory deposits which are permanent.

\section{Maintaining cause}

- Damp dwelling

- Persistent mental worries and anxiety

- Over use and improper use of various joints as seen occupational habits

- Obesity

\section{Exciting causes}

- Occupational

- Environmental

- Physical exertion

- Emotional

\section{Predisposing factors}

- Age: more than 40 years of age

- Sex: female
- Season: wet rainy weather

- Vaccination

\section{Few drugs used in rheumatoid arthritis}

- Though homoeopathy has no specific remedy for Rheumatoid arthritis, but it has proved to be of par excellence in curing these cases based on symptom similarity.

- Abrotanum: Rheumatism following checked Diarrhœa. Pain in shoulders, arms, wrists, and ankles. Pricking and coldness in fingers and feet. Legs greatly emaciated. Joints stiff and lame. Painful contraction of limbs.

- Arnica montana: Chronic arthritis with a feeling of bruising and soreness may be helped by this remedy. The painful parts feel worse from being moved or touched.

- Aurum metallicum: Wandering pains in the muscles and joints that are better from motion and warmth, and worse at night, suggest a need for this remedy.

- Bryonia alba: This remedy can be helpful for stiffness and inflammation with tearing or throbbing pain, made worse by even the smallest motion.

- Calcarea carbonica: This remedy may be useful for deeply aching arthritis involving node formation around the joints. Inflammation and soreness are worse from cold and dampness, and problems may be focused on the knees and hands.

- Calcarea fluorica: This remedy is often indicated when arthritic pains improve with heat and motion. Joints become enlarged and hard, and nodes or deformities develop. Arthritis after chronic injury to joints also responds to Calcarea fluorica.

- Colchicum autumnale: Sharp pain down left arm. Tearing in limbs during warm weather, stinging during cold. Pins and needles in hands and wrists, fingertips numb.

- Ruta graveolens: Arthritis with a feeling of great stiffness and lameness, worse from cold and damp and worse from exertion, may be helped with this remedy. Tendons and capsules of the joints can be deeply affected or damaged. The arthritis may have developed after overuse, from repeated wear and tear.

- Rhus toxicodendron: Rheumatoid arthritis, with pain and stiffness that is worse in the morning and worse on first motion, but better from continued movement, may be helped with this remedy. Hot baths or showers, and warm applications improve the stiffness and relieve the pain.

- Ledum palustre: Arthritis that starts in lower joints and extends to higher ones may respond to this remedy. Pain and inflammation often begin in the toes and spread upward to the ankles and knees.

\section{Repertorial approach in rheumatoid arthritis Pain rheumatic (Kent repertory)}

$1^{\text {st }}$ Grade: Arn, Ars, Aur-m-n, Bad, Bez-ac, Bry, Caust, Cham, Chel, Colc, Form, Kali-I, Kalm, Lyco, Medo, Nat-a, Phyt, Puls, Rhod, Rhus-t, Sang, Sarc, Sulph.

Pain rheumatic right to left: Lyco

Pain rheumatic left to right: Lach, Rhus-t

Pain acute: Acon, Bryo, Colch, Merc, Rhus-t

Pain rheumatic alternating with: 
Gastric symptoms: Kali-bi

Diarrhoea: Kali-bi

Pulmonary trouble: Kali-bi

Pain rheumatic Cold after a:

Amel: Led, Puls, Sec

Becoming: Rhut-t

Weather: Bryo, Calc-p, Rhus-t

Drive him out of bed: Cham, Merc

Gonorrhoea after suppressed: Medo, Thuj

Mercury abuse of: Chin, Guaj, Hep, Sarc.

Perspiration with: Form-ac, Merc

Syphilitic: Kali-i

Weather cold: Bryo, Calc-P Rhus-t

Worm weather in: Colch

Extending upward: Led

Sitting: Valer

Thunderstorm agg: Med, Rhus-t

Touch agg: Chel

Walking on amel: Rhus-t

Wandering, sifting: Amn-m, Carb-s, Kali-s, Lac-c, Puls

Warmth agg; Sec

Amel: Ars, Kali-bi, Kali-p, Mag-p, Rhus-t, Sil

Of bed agg: Merc

Amel: Ars, Rhus-t

Wet weather agg: Calc, Colch, Merc, Puls, Rhod, Rhus-t Verat

Pain joint rheumatic:

Aur, bry, Calc-p, Caust, Colch, Fer-p, Form, Iod, Kali-bi, Lyco, Rhus-t,

Spig

Gonorrhoea after suppressed: Med, Thuj

Sour wine, after: Ant-c

Wandering: Aur, Kali-bi, Lac-can, Puls

Warmth agg: Led, Puls

Amel: Ars

Warmth of bed agg: Led

Pain upper limb, rheumatic: Bryo, Calc-p, Colch, Ferr, Merc, Rhus-t, Sang

Shoulder, rheumatic: Colc, Ferr, Med, Rhod, Rhus-t, Sulph

Upper arm, rheumatic: Ferr, Rhus-t, Sang

Wrist, rheumatic: Ruta, Rhus-t.

Pain, Hand, Rheumatic: Caul, Colch, Rhus-t

Fingers, rheumatic: Caul

Pain lower limb, rheumatic: Led

Hip, rheumatic: Colch, Rhus-t

Knee, rheumatic: Bryo, Calc, Kali-c, Rhus-t.

Foot, rheumatic: Hep, Led.

Heel, rheumatic: Rhod

Toes, rheumatic: Aur

\section{Conclusion}

Rheumatoid arthritis is not away from the Homoeopathy law. The doctrine of symptom similarity and derangement of vital force cured by internal medicine. Homoeopathy depends on Proper case taking and individualization and framing the totality of symptoms.

We don't have much scope in surgical cases but we have a better way of treatment in non-surgical cases. So, in this century the holistic approach is the nest way of treating a case of osteoarthritis. We must stick to our fundamental principle Similia Similibus Curantur which helps as curative and preventive. So, in this type of cases homoeopathy offers a great scope of treatment in a most harmless and proper way.

\section{References}

1. Harrison's $15^{\text {th }}$ edition - principal of internal medicine.

2. Davidson's $18^{\text {th }}$ edition - principles and practice of medicine.

3. Munjal YP. API textbook of medicine, $10^{\text {th }}$ edition, Mumbai, Jaypee brother medical publisher (p) ltd 2015, 1 .

4. Kundu Arup Kumar. Bedside clinics in medicine. Second edition. Kolkata: Academis publishers 1997.

5. Bailey and love's, short practice of surgery - edited, Mass Williams, Russell $-22^{\text {nd }}$ edition.

6. Text book of pathology $3^{\text {rd }}$ edition - Harsh Mohan.

7. Arthritis by. Reddy E, Sharma PK, Raj PP. A clinical study on effect of Plantago in gingivitis by assessing bleeding and plaque index. Dr. Ritu Arora.

8. Organon of medicine by R.E. Dudgeon.

9. Homoeopathic therapeutics by Samuel Lilienthal.

10. The principles and art of cure by homoeopathy Herbert A. Roberts.

11. William Boericke. Boerickes new manual of homoeopathic materia medica with repertory, LPE series, $14^{\text {th }}$ impression 2016.

12. The chronic miasms $0 \mathrm{~J}$ Henry Allen.

13. Allen HC. Keynote and characteristics with comparison of some of the leading remedies of the materia medica with bowel nosode, B Jain publishers (p) ltd., New Delhi, $08^{\text {th }}$ edition.

14. Hahnemann Samuel. Organon of medicine, 5th \& 6th edition, Kolkata, modern homoeopathic publishers 2013-2014.

15. Kent JT. Repertory of homoeopathic materia medica. Enriched Indian edition reprint from sixth American edition. Published by b. Jain publishers (p) ltd 2017. 